\title{
A Comparative Studyon Kernel PCA and PCA Methods for Face Recognition
}

\author{
Mohammad Mohsen Ahmadinejad ${ }^{1}$, Elizabeth Sherly² \\ ${ }^{1}$ Department of Computer Science University of Kerala , India \\ ${ }^{2}$ School of Computer Science \& Information Technology, IIITM-K, Kerala, India
}

\begin{abstract}
A online face recognition system is a dynamic topic in the fields of biometrics, which Many achievements have been obtained in face recognition.The human face has a principal role which,is consists of complicated combination of features that allows us to communicate, express our feelings and emotions. Principal Components Analysis (PCA) and kernel principal components, Analysis (KPCA) are achievements that, have been obtained in face feature extraction and recognition. In this papar have compared a PCA algorithm with KPCA algorithm, which a LFW data set is used for Compare, Recognition accuracy, Variation in Facial Expression, Illumination changes, and Computation time of each method. To find Recall of each algorithm are used a LFW database which shows a 2D-PCA have better performance.
\end{abstract}

Keywords: KPCA, PCA, algorithm, comparative,error rates

\section{Introduction}

Most of the face recognition systems can recognize human faces automatically which are uses in security, cyberspace, etc. But a robust face recognition system in an unconstrained area should detect and recognize any face trough different pose, illumination and expression. Industries are looking for the system with more accurate and error free face recognition systems to meet the complexity of practical scenarios. Automated face recognition is a relatively new concept. First semi-automated system proposed for a face Recognition system in the 1960, which required the locate features of face such as eyes, ears, nose, and mouth then are calculated distances and ratios to a common point, which then compared each data. Goldstein, Harmon et. al are used 21 specific subjective markers such as hair color and lip thickness to a automatic face recognition system In the 1970s. In 1988, Kirby and Sirovich applied principle component analysis, a standard technique to the a face recognition system In the 1970s, , Turk and Pentland proposed a face recognition system that using the eigenfaces techniques, thuse the residual error could be used to detect faces in images in 1991. A face recognition system can automatically verifyor identify a person from a digital figure or a video frame. In most of face recognition algorithms identify faces images distinctivest by extracting face feature, landmarks, etc. from an image which includes face. A person can be identified by other means than the face such as Voice, body shape, etc. But, a face part is more distinctive key to a person's identity. Anyway automatic [1] face recognition contains three problems of 1..Detection and rough normalization of faces which This is determined, whether human faces appear in a given image, and where faces are located 2. Feature extraction and accurate normalization of faces that explain after the face detection step, face image patches are extracted from images 3 . Identification or verification, which contain two primary tasks, first is verification one-to-one matching key-points when presented with a unknown face image and Second is identification of face.
The PCA classifier is one of the methods for classification which, involves a mathematical course of action that transform a possible correlatenumber of variable into a sminor uncorrelatenumber of variable which, called principle component that aPCA is mathematically defined as a linear transform that transform the data to a new coordinate system such that best variance which achives by projection of the statistics which, comes to lie on the first coordinate.A PCA algorithm computes means, covariance, variances, and [2] correlations of large data sets.

Traditional PCA only allows linear dimensionality [3] reduction but, Kernel PCA allows us to generalize linear PCA to nonlinear dimensionality reduction.AKernel PCA algorithm is a nonlinear form of PCA, which works better in complicated spatial structure of high-dimensional features.

\section{Related Work}

A face recognition system is a dynamic topic of biometrics, which automatically identify or verifies a person from a digital figure or a video frame. So some of the face recognition algorithms identify faces by extracting features or landmarks, of an image.Kernel principal components Analysis (KPCA) is an elementary technique widely used in feature extraction and recognition of a face image, which is based on PCA method.

J Zhang et al. (1997)they survey and compare three algorithms for face recognition: classificationand eigenface, autoassociation a neural nets, so they analyzed under a common statistical decision framework, then they used four different databases, which have moderate subject size and a combined database with different subjects for evaluated each algorithm. So experimental result, indicate eigenface algorithm that has essentially a minimum distance classifier when lighting variation is small [4].

Guo, Guodong, Stan Z. Li, and Kapluk Chan. (2000) proposed a SVM as a new technique for pattern recognition, whichSupport vector machines used with a binary tree 


\section{International Journal of Science and Research (IJSR) \\ ISSN (Online): 2319-7064}

Index Copernicus Value (2013): 6.14 | Impact Factor (2015): 6.391

recognition strategy to tackle the face recognition problem. An ORL face database, which consists of 400 images of 40 individuals, is used to evaluate performance of new method. They also present an experiment for recognition on a larg face database. Then theycompare the SVM-based recognition with the standard eigenface approaches using the nearest center classification (NCC) criterion[5].

Kim et al.(2002) they have presented a Kernel PCA based face feature extraction method, they used polynomial kernel principal components to compute the product space of input pixels which making up a facial pattern. To show the effectiveness of the proposed method an SVM method have used as the recognition and an ORL database used[6].

Ganet al.(2005)in In their research, is presented with the advantages of PCA, and an improved method 83 Jun-ying Gan, Dang-Pei Zhou, Chun-Zhi Li based on the normalization of within-class average face image. Then they have compared with traditional PCA method, which their result has shown moreacceptable to process samples with different class and same class.Therefore, this experimental showswhich, a higher correct recognition rate can be acquired, then a better efficiency can be achieved[7].

Timotius et al.(2010)In their paper a face recognition method based on the combined KPCA analysis kernel principal component and support vector machine SVM methods have presented, that KPCA method is utilized to extract features from the input images,then SVM method is applied to classify the input images. They compare the performance of this face recognition method to other commonly-used methods which their experiments show that the combination of KPCA and SVM achieves a higher performance compared SVM, and the combination of kernel principal component analysis[8].

Ebied, Rala M. et al. (2012) In their studies have shows use of linear and nonlinear methods for feature extraction in the face recognition system. Widely The linear PCA used in the face recognition is used to construct the feature space and extract features. A KPCA is extended from PCA to a nonlinear mappings in a higher dimensional feature space. In Kernel function several parameters has investigated and expected to affect the recognition performance. Thus the knearest neighbor classifier with Euclidean distance is used in the classification step. In their experiments used a ORL face database which contains variability in expression, pose, and facial details then, results show that KPCA with Gaussian function can give a correct recognition rate similar to PCA and higher than Kernel-PCA with polynomial function[9].

Upadhayay, Ritu, and Rakesh Kumar Yadav(2013) in Their surveys presents nitty gritty of KPCA and an up to date review of techniques KPCA. They notify benefits of KPCA over PCA. Finally, theyfind that it isa good appropriate technique forface recognition systems. Therefore, they shows with reviewing existing face recognition system a KPCA for face recognition system can have a highest recognition[10].

\section{PCA Method}

The principal component analysis classifier is linear method to classification which transform a number of possible variable which are correlated into a smaller number of variable which are uncorrelated called principle component, that is mathematically defined as an linear transform that transform the data to a new coordinate system. A principal component analysis computes means, covariance, variances and correlations of large data sets. PCA computes and ranks principal components and their variances.

Thuse as benefits of aprincipal component analysismethod are, reduce the dimension of the data, complexity of the images can be reduced.This years the number of extention of pca is used such as,1.Improved PCA, 2. Fuzzy PCA, 3.Incremental PCA ,4. Kernel PCA.

\subsection{Improved PCA}

Usually in PCA face image matrix must be converted into a high dimensional vector matrix, thuse directly is verydifficult to process and recognize the face image matrix but improved PCA, Original face image can be represented as a two dimensional matrix.

\subsection{Fuzzy PCA}

A Fuzzy PCA has used to resolve problem of a PCA which a PCA method does not always show the real similarities structure on the data in the higher dimensional space. So a fuzzy PCA used to get data into more feasible form.

\subsection{Incremental PCA}

In existing PCA based face recognition systems because of the cost and memory requirement burden are hard to scale up. An incremental approach is usually adopted for resolve this limitation.

\subsection{Kernel PCA}

A KPCA method allows alinear PCA method to nonlinear dimensionality reduction. PCA method only allows linear dimensionality reduction thus, data that are complex, and cannot be simplified in a linear subspace a PCA method will become invalid and because of this limitation a kernel PCA method has developed. Although a KPCA is a development form of the PCA method.

\section{PCA Process}




\section{International Journal of Science and Research (IJSR) \\ ISSN (Online): 2319-7064}

Index Copernicus Value (2013): 6.14 | Impact Factor (2015): 6.391

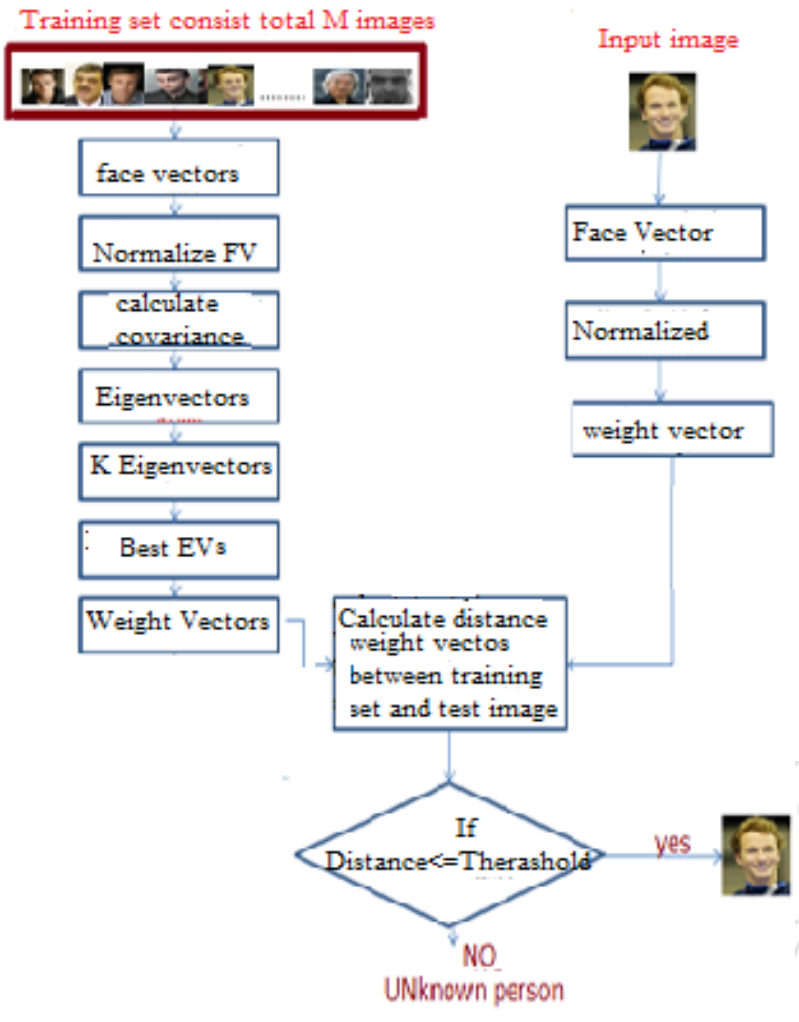

Figure 1: PCA process for face recognition

\section{PCA Steps}

5.1 Subtract the mean from all the data points

Once we have created a training set then all images in training set converts each to vector column. Thuse, If image presents by the size of $N * N$ converts to a $1 * N^{2}$ vector column form. Once we have converted all images to face vectors, the will remove all the common features that all images share together (normalization), because each face in left behind must be with unique futures .In the normalization calculates the average vector (Mean) and subtract from face vectors , mathematically calculate the Mean of each observation that is shown in equation 1 .

$$
M i=\sum_{i=0}^{m n} \sum_{i=1}^{p} \text { face_Db(i,j) }
$$

\subsection{Compute the covariance matrix and Calculate eigenvectors}

The covariance matrix has given by bellowing formula that Calculate high dimensional in equation 2 .

$$
\begin{gathered}
\mathrm{C}=\mathrm{A} A^{T} \\
\text { Where } \mathrm{A}=\left\{\mathrm{N}_{f 1}, \mathrm{~N}_{f 2}, \mathrm{~N}_{f 3}, \ldots \ldots \ldots \ldots \mathrm{N}_{f m}\right\}
\end{gathered}
$$

By calculate above Covariance matrix a huge matrix with size of $\mathrm{C}=A_{N^{2} * M} \cdot A^{T}{ }_{M * N^{2}}$ thus $C_{N^{2} * N^{2}}$ produces.

\subsection{Reduce high dimensional eigenvector matrix to low dimensional eigenvector matrix}

With a high dimensional eigenvector matrix system will run slowly or run out of memory because the computational is so
huge,So The solution is dimensionaly reduction. Reduce high dimensional eigenvector matrix to low dimensional eigenvector matrix which the formula as shown in equation 3.

$$
\mathrm{C}=A^{T} \mathrm{~A}
$$

Then the result is one low dimensional eigenvector matrix with size of $\mathrm{C}=A_{M * N^{2}} \cdot A^{T}{ }_{N}^{2} * \mathrm{M}$ thus $C_{\mathrm{M} * \mathrm{M}}$.

\subsection{Selected best K Eigen-Faces and calculate Weight vector}

A selected $\mathrm{K}$ Eigen-Faces should be in the original dimensionality of the face vector space, but we find the significance K Eigen-Faces in low dimensional eigenvectors, then for represent the original dimensionality we have to move back to high dimension eigenvector which is done by below formula.

$\mathrm{Vi}=$ high dimensional eigenvector

$\mathrm{Ui}=$ low dimentinal eigenvector

$\mathrm{Ui}=\mathrm{AVi}$

For finding weight vector The weight vector represents by PCA result.Each $w$ is the percentage of each eigenface that contribute to make each image in training set

\section{Kernel PCA Process}

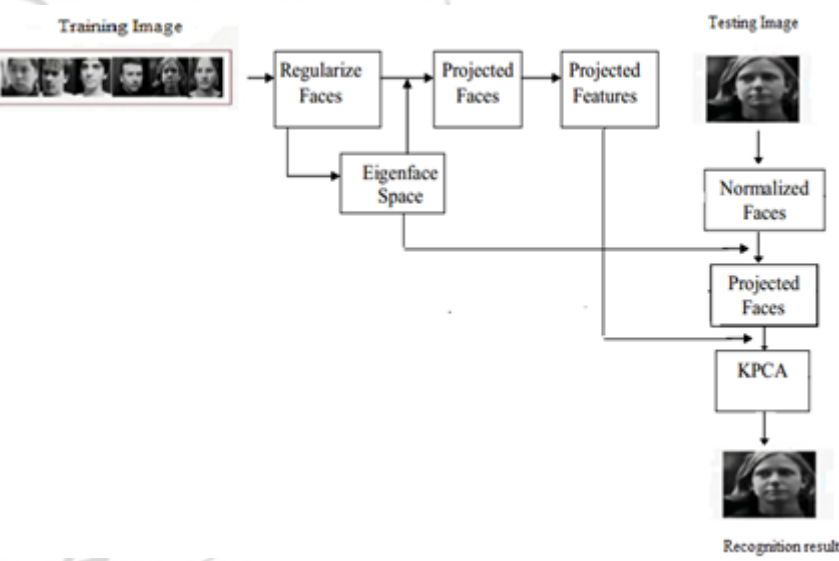

Figure 2: Kernel PCA process.

\section{The Standard Kernel PCA Steps}

7.1 Construct the kernel matrix $K$ from the training data set (xi) which shows in equation 4 .

$$
K i, j=K(x i, x j) .
$$

7.2 Compute the Gram matrix Ke using Equation 5.

$$
\widetilde{\mathbf{K}}=\mathrm{k}-1 \mathrm{Nk}-\mathrm{k} 1 \mathrm{~N}+1 \mathrm{~N} \mathrm{~K} 1 \mathrm{~N}
$$

7.3 solve the vectors ai (substituteK with Ke ) use the equation 6.

$$
\mathbf{K} \mathbf{a}_{k}=\lambda_{k} N \mathbf{a}_{k}
$$




\section{International Journal of Science and Research (IJSR) \\ ISSN (Online): 2319-7064}

Index Copernicus Value (2013): 6.14 | Impact Factor (2015): 6.391

7.4 Compute the kernel principal components use equation 7

$$
y_{k}(\mathbf{x})=\phi(\mathbf{x})^{\mathrm{T}} \mathbf{v}_{k}=\sum_{i=1} a_{k i} \kappa\left(\mathbf{x}, \mathbf{x}_{i}\right)
$$

\section{Compare the Error Rates Between PCA and Kernel PCA}

For finding the error rate between PCA and KPCA the Yale database has used that, 4860 single light source of 10 different subjet with different views has used. The result which shoes error rat has shown in table 1 .

Table1: Error rate in training and testing data

\begin{tabular}{|c|c|c|}
\hline Error rate & Training data & Testing data \\
\hline PCA & $9.35 \%$ & $25.32 \%$ \\
\hline KPCA & 7.90 & $13.56 \%$ \\
\hline
\end{tabular}

\section{Result}

To find Recall of each algorithm are used a Yela database which the result are shown in table 2 .

Table2: compartive SIFT, 2D-PCA, PCA,KPCA

\begin{tabular}{|c|c|c|c|c|}
\hline Method /Performance & SIFT & 2D-PCA & PCA & KPCA \\
\hline Recognition accuracy & Good & Good & Good & Good \\
\hline $\begin{array}{c}\text { Variation in Facial } \\
\text { Expression }\end{array}$ & poor & Good & Normal & Good \\
\hline Illumination Changes & poor & Good & Normal & Normal \\
\hline Computation time & Normal & Normal & Good & Good \\
\hline
\end{tabular}

\section{Conclusion}

In this paper compared three different algorithms which used the LFW AND Yela datasets for face recognition. For rate of error in traing set and test data which, used Yela database has shown that KPCA with less error have better result .The recognition accuracy, Variation in Facial Expression, Illuminationchanges, Computation time are compared for each algorithms with Yela data set that shows a KPCA for face recognition have better performance.

\section{References}

[1] W. Zhao R. Chellappa P. J. Phillips , "Face Recognition: A Literature Survey", ACM Computing Surveys , 2003, Vol. 35, Issue 4, Pp: 399-458

[2] Srinivasulu Asadi, Dr.Ch.D.V.Subba Rao, V.Saikrishna, "A Comparative study of Face Recognition with Principal Component Analysis and Cross-Correlation Technique", IJCA, 2010, Vol.10, No.8, PP: 0975 - 8887

[3] Quan Wang, "Kernel Principal Component Analysis and its Applications in Face Recognition and Active Shape Models", 2012.

[4] Zhang, Jun, Yong Yan, and Martin Lades. "Face recognition: eigenface, elastic matching, and neural nets." Proceedings of the IEEE 85.9 (1997): 1423-1435.

[5] Guo, Guodong, Stan Z. Li, and Kapluk Chan. "Face recognition by support vector machines." Automatic
Face and Gesture Recognition, 2000. Proceedings. Fourth IEEE International Conference on. IEEE, 2000.

[6] Kim, Kwang In, Keechul Jung, and Hang Joon Kim. "Face recognition using kernel principal component analysis." Signal Processing Letters, IEEE 9.2 (2002): 40-42.

[7] Gan, Jun-ying, Dang-pei Zhou, and Chun-zhi Li. "A method for improved PCA in face recognition." International Journal of Information Technology 11.11 (2005): 79-85.

[8] Timotius, Ivanna K., Iwan Setyawan, and Andreas A. Febrianto. "Face recognition between two person using kernel principal component analysis and support vector machines." International Journal on Electrical Engineering and Informatics 2.1 (2010): 55-63.

[9] Ebied, Rala M. "Feature extraction using PCA and Kernel-PCA for face recognition." Informatics and Systems (INFOS), 2012 8th International Conference on. IEEE, 2012.

[10] Upadhayay, Ritu, and Rakesh Kumar Yadav. "Kernel principle component analysis in face recognition system: a survey." International Journal of Advanced Research in Computer Science and Software Engineering 3.6 (2013): 348-353.

\section{Autor Profile}

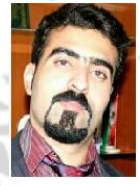

MohammadMohsen Ahmadinejad, took his Post Graduation in Scientific computing in an interdisciplinary school of scientific computing, University of Pune in 2010. He has two years of teaching experience after the Post Graduate level and four Years experience in the research field. $\mathrm{He}$ is currently pursuing his Ph.D. in Online face recognition in unconstrained environments, University of Kerala, India. Her research interests include online methods for face recognition and methods for extract face features.

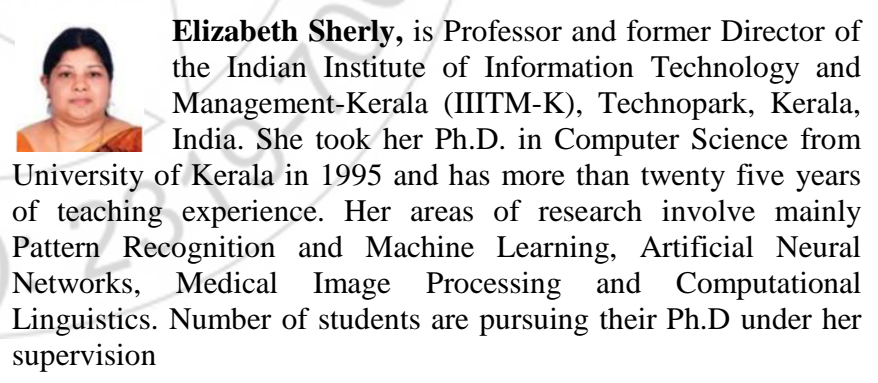

Elizabeth Sherly, is Professor and former Director of the Indian Institute of Information Technology and Merala (IIITM-K), Technopark, Kerala,

India. She took her Ph.D. in Computer Science from University of Kerala in 1995 and has more than twenty five years of teaching experience. Her areas of research involve mainly Pattern Recognition and Machine Learning, Artificial Neural supervision 